\title{
Euclidean Quantum Fields with Spin on an Indefinite Inner Product State Space
}

\author{
By
}

Bengt $\mathrm{EK}^{*}$

\begin{abstract}
It is shown that for a Wightman quantum field theory with space inversion symmetry, the Wightman functions in Euclidean points can be obtained as certain linear combinations of vacuum expectation values of Euclidean fields, on a state space with an indefinite inner product. The coefficients of these linear combinations only depend on the transformation properties of the fields, not on their interactions.

The use of an indefinite inner product enables us to keep the number of Euclidean fields the same as that of Wightman fields, except that in the case of hermitian fermion fields, the corresponding Euclidean fields are not hermitian.

For free Dirac fields, the construction is exhibited explicitly and a Euclidean invariant Hilbert space metric on the state space, making the indefinite inner product continuous, is introduced.
\end{abstract}

\section{$\S 1$. Introduction}

The Euclidean approach to quantum field theory (i.e. the study of the vacuum expectation values at imaginary times) is an attempt to simplify the problems by going over to totally commuting or anticommuting fields. (For the properties of the Euclidean Green's functions and references to early work in the field, see Osterwalder-Schrader, $[1,2]$ ).

By utilizing the fact that for many models the Euclidean Green's functions, or Schwinger functions, can be obtained as vacuum expectation values of $\mathrm{Eu}-$ clidean fields, much progress has been achieved in constructive quantum field theory. In the case of bosons one can use probabilistic methods (see e.g. Nelson [3]), and for fermions their anti-commutative analogues (e.g. Gross [4]).

Communicated by H. Araki, February 23, 1981.

* Research Institute for Mathematical Sciences, Kyoto University, Kyoto 606, Japan. Present address: Dept. of Theoretical Physics, Royal Institute of Technology, S-100 44 Stockholn, Sweden. 
In [5] Osterwalder and Schrader construct Euclidean fermion fields corresponding to the free Dirac field. They find that since the Wightman functions are not hermitian in Euclidean points, they must introduce extra independent fields. I.e. adjoint relativistic fields do not correspond to adjoint Euclidean fields in their formulation, the "number of degrees of freedom" is increased. Özkaynak [6] has studied free fields of arbitrary spin in a similar way.

Fröhlich and Osterwalder in [7] avoid the doubling of the number of fields in a certain (weak) sense. However, they conclude that it seems difficult to construct interacting fermion fields in a natural way.

In the present paper, it is shown that if we allow the inner product on the Euclidean state space to be indefinite, we can avoid the introduction of extra fields (except in the case of hermitian fermion fields, which will correspond to non-hermitian Euclidean fields) if we restrict ourselves to interactions that preserve parity. Explicitly, for a Wightman theory, in the sense of StreaterWightman [8], with space inversion invariance we construct Euclidean covariant fields acting on an (indefinite) inner product space, such that their "vacuum", expectation values are linear combinations (independent of the interactions) of the Wightman functions in Euclidean points. These fields commute (anticommute for pairs of fermion fields) in all points and adjoints in the relativistic theory correspond to adjoints with respect to the inner product.

It is clear that the last two properties exclude a definite inner product for Euclidean fermion fields (in Hilbert space, no non-zero operator anti-commutes with its own adjoint).

The inner product of our construction is uniquely determined by the relativistic theory except for the non-uniqueness of the extensions of the Schwinger functions to Euclidean points of coinciding arguments. For what interactions the extensions can be chosen to give a definite inner product for a theory involving bosons only, seems to be a non-trivial question (cf. $[9,10]$ ).

Except in the proof of Lemma 2 below, our proof does not explicitly use the positivity of the inner product in the relativisitc state space.

Simon [11] has pointed out the connection between Nelsons axioms for free fields and space inversion invariance. Borchers and Yngvason [12] mention the connection with the reality of the measure in a probabilistic formulation.

Following Feinberg and Weinberg [13], we allow the phase factors in the space inversion operation to be arbitrary.

We note that any field theory can be embedded in one with space inversion 
invariance (by doubling the number of fields).

An indefinite inner product does, of course, not in general define a normed topology. In the case of the free Dirac field (which is treated in detail below, as an introduction to the general case) we will see that there is a majorizing (in the sense of Bognár [14]), invariant positive definite inner product on the state space. This brings us close to the formulation of [5]. Whether we can always find such a majorizing inner product by choosing our extension of the Schwinger functions properly is not clear (cf. [15]).

It seems (at least to the author) natural to consider the indefinite inner product structure more intrinsic, and say that we can obtain a Hilbert space formulation analogous to that in [5] only when a positive definite majorant exists.

We do not treat the question of Markov properties of the fields (cf. $[3,16]$ ), nor the (important) problem of conditions on the Euclidean theory to correspond to a Wightman theory in the relativistic region (some conditions are of course direct from [2]).

For a description of different approaches to Euclidean Fermi fields and their relationships, see Palmer [17].

The organization of this paper is as follows. After this introduction, we present our main results in Section 2. These results are proved in Section 3 for the free Dirac case and in Section 4 for the general case. In these sections, more precise formulations of our assumptions and notation are given.

\section{§2. Main Results}

We first restrict ourselves to the case of the free Dirac field. This means that we have explicit expressions for the Schwinger functions and it is easy to follow the manipulations. Proofs of Lemma 1 and Propositions 1 and 2 can be found in Section 3.

Let $\mathbb{\Xi}^{0}(x, y)$, defined for $x, y \in \mathbb{R}^{4}$ such that $x \neq y$, be the $4 \times 4$ matrix Schwinger functions for the free Dirac field (see eq. (3.8)).

Lemma 1. There exists an invertible $4 \times 4$ matrix $\mathbb{C}$ such that

$$
\mathfrak{t}^{0}(x, y)=\mathfrak{s}^{0}(x, y) \mathbb{C}^{*}
$$

is hermitian:

$$
\mathfrak{X}^{0}(x, y)=\mathfrak{X}^{0}(y, x)^{*}
$$


Such a $\mathbb{C}$ is unique up to multiplication with a non-zero real number.

Here $A^{*}$ denotes the hermitian adjoint of the matrix $\mathbf{A}$.

The integral representation (3.14) of $\mathfrak{X}^{0}(x, y)$, originally defined for $x \neq y$, gives natural extensions to distributions on the Schwartz functions $\mathscr{S}\left(\boldsymbol{R}^{8}\right)$. They will also be denoted $\mathfrak{x}^{0}(x, y)$.

Definition. These $\mathfrak{X}^{\circ}$ and the many point functions obtained from their products will be called the redefined Schwinger functions (for the free Dirac field).

Proposition 1. There exists a non-degenerate indefinite inner product, complex vector space $(\mathscr{K},\langle\cdot, \cdot\rangle)$ with a vacuum vector $\Omega$, a unitary representation $\mathrm{V}(\mathrm{A}, \mathrm{B}, a)$ of the covering group of the inhomogeneous Euclidean group, and four component fields $\Psi, \Psi^{\dagger}$ (i.e. operator-valued distributions on $\mathscr{S}\left(\boldsymbol{R}^{4}\right)$ with values acting on all of $\left.\mathscr{K}\right)$, such that

$\Omega$ is invariant under $\mathrm{V}(\mathrm{A}, \mathrm{B}, a)$ and (algebraically) cyclic w.r.t. $\Psi, \Psi^{\dagger}$,

$\Psi$ transforms covariantly under V(A, B, a) (see (3.28-29)), for all $x, y$ $\in \mathbb{R}^{4}, \Psi$ satisfies the following relations in distribution sense:

$$
\left\{\Psi(x), \Psi^{\dagger}(y)\right\}=\{\Psi(x), \Psi(y)\}=\left\{\Psi^{\dagger}(x), \Psi^{\dagger}(y)\right\}=0,
$$

the vacuum expectation values of $\Psi, \Psi^{\dagger}$ are the re-defined Schwinger functions and

$\Psi^{\dagger}$ is the adjoint of $\Psi$ w.r.t. $\langle\cdot, \cdot\rangle$.

The covering group of the inhomogeneous Euclidean group (in four dimensions) is a semi-direct product of $S U(2) \times S U(2)$ and $\mathbb{R}^{4}$, i.e. $\mathrm{A}, \mathrm{B} \in S U(2)$ and $a \in \mathbb{R}^{4}$ above.

Unitarity of $\mathrm{V}$ is with respect to $\langle\cdot, \cdot\rangle$, i.e. $\mathrm{VV}^{\dagger}=\mathrm{V}^{\dagger} \mathrm{V}=\mathbb{1},\{\cdot, \cdot\}$ denotes the anti-commutator.

The next result actually provides a relation between our work and that of $[5]$.

Proposition 2. On $\mathscr{K}$ of Proposition 1 there exists a positive definite inner product $(\cdot, \cdot)$ such that $(\Omega, \Omega)=1, \mathrm{~V}(\mathrm{~A}, \mathrm{~B}$, a) is unitary w.r.t. $(\cdot, \cdot)$ and the topology induced by $(\cdot, \cdot)$ majorizes $\langle\cdot, \cdot\rangle$, i.e. $\langle\cdot, \cdot\rangle$ is (jointly) continuous in that topology.

Remark 1. Concerning the uniqueness of a $(\cdot, \cdot)$ with the properties in Proposition 2, see the discussion in Remark 1 at the end of Section 3. 
Remark 2. Although the relativistic theory is space inversion invariant, the Euclidean one is not. See Remark 2 at the end of Section 3.

We now come to the more general case of an interacting Wightman theory. The proofs of the following results are in Section 4, with more detailed definitions.

Consider a field theory (as defined in Chapter 3 of [8]) with space inversion symmetry. We have, then, a unitary representation $U$ of the orthochronous Poincaré group $\mathscr{P}^{\uparrow}$ (i.e. preserving time directions) and a finite number of linearly independent fields $\psi_{\gamma}(x)$ acting on some Hilbert space. By $(4.1,2)$ and (4.4) the fields transform among themselves under $U$ and under taking adjoints.

To construct the re-defined Schwinger functions we need the following

Lemma 2. The linear basis for the fields above can be chosen to be $\left\{\psi_{\alpha_{i}}^{(i)}\right\}_{i \in \mathcal{S}}$ with the finite range of $\alpha_{i}$ depending on $i$ and each $\psi^{(i)}$ transforming by an irreducible representation of the covering of the orthochronous Lorentz group $\mathrm{L}^{\uparrow}$. Also, the finite index set $\mathscr{I}=\mathfrak{I} \cup \mathfrak{F} \cup \mathfrak{n}$ disjointly, where

$$
\begin{gathered}
\mathrm{n}=\left\{i \in \mathscr{I} \mid \psi^{(i)^{*}}=\psi^{(i)}\right\}, \\
\mathfrak{f}^{*}=\left\{i \in \mathscr{I} \mid \psi^{(i)^{*}}=\psi^{(j)}, \text { some } j \in \mathfrak{I}\right\},
\end{gathered}
$$

and $\psi^{(i)^{*}}$ is the field with components $\left(\psi_{\alpha}^{(i)}\right)^{*}$.

In the following, we assume the fields chosen according to Lemma 2, but also extend the index set $\mathscr{I}$ by introducing formal adjoints $i^{*}$, such that $\psi^{\left(i^{*}\right)}$ $=\psi^{(i)^{*}}$, and by distinguishing $i$ and $i^{*}$ for hermitian fermions (but not for hermitian bosons). Accordingly, $n$ will from now on (except in Subsection 4.A, the proof of Lemma 2) only contain indices for hermitian boson fields. We will construct Euclidean fields which are indexed by this extended $\mathscr{I}$.

If $\mathrm{R}=i_{1}, \ldots, i_{n}$ is a sequence of indices in $\mathscr{I}$, let $\mathrm{R}^{*}$ denote the sequence $i_{n}^{*} \cdots i_{1}^{*}$ and $\mathfrak{B}_{\mathrm{R}}\left(z_{1}, \ldots, z_{n}\right)$ be the Wightman functions given by analytic continuation in the arguments of the vacuum expectation values of $\psi^{\left(i_{1}\right)}\left(x_{1}\right) \cdots$ $\psi^{\left(i_{n}\right)}\left(x_{n}\right)$ (cf. [8]).

If $\pi \in \mathscr{P}_{n}$ (the symmetric group of degree $n$ ), $\pi(\mathrm{R})$ is the sequence $i_{\pi^{-1}(1)} \cdots$ $i_{\pi^{-1}(n)}$ and $\sigma(\pi, \mathrm{R})= \pm 1$ according as the number of transpositions of fermi indices in $\mathrm{R}$ by $\pi$ is even or odd.

The Schwinger functions $\mathfrak{S}_{\mathrm{R}}\left(x_{1}, \ldots, x_{n}\right)$ are defined to be $\mathfrak{B}_{\mathrm{R}}\left(x_{1}^{\prime}, \ldots, x_{n}^{\prime}\right)$ for distinct $x_{i}$ in $\mathbb{R}^{4}$, where $a^{\prime}$ for $a=\left(a_{0}, a_{1}, a_{2}, a_{3}\right)$ is defined by 


$$
\left(a_{0}, a_{1}, a_{2}, a_{3}\right)^{\prime}=\left(i a_{0}, a_{1}, a_{2}, a_{3}\right) .
$$

The $\alpha$-indices of $\mathfrak{W}_{\mathrm{R}}, \mathfrak{\subseteq}_{\mathrm{R}}$ etc. will not be written out, but summation over them will sometimes be understood.

Proposition 3. For each sequence $\mathrm{R}$ of indices from $\mathscr{I}$, there exists an invertible constant matrix $\mathbf{C}_{R}$ (acting on the $\alpha$-indices of $\mathfrak{\Xi}_{R}$, see (4.33)) such that the (restricted) redefined Schwinger functions

$$
\mathfrak{t}_{\mathrm{R}}\left(x_{1}, \ldots, x_{n}\right)=\mathbf{C}_{\mathrm{R}} \Im_{\mathrm{R}}\left(x_{1}, \ldots, x_{n}\right)
$$

are hermitian:

$$
\mathfrak{\mathfrak { t }}_{\mathrm{R}}\left(x_{1}, \ldots, x_{n}\right)=\overline{\mathfrak{\mathfrak { t }}_{\mathrm{R}^{*}}\left(x_{n}, \ldots, x_{1}\right)}
$$

(the bar denotes complex conjugation).

The $\mathfrak{X}_{\mathrm{R}}$ are real-analytic functions defined for

$$
\left(x_{1}, \ldots, x_{n}\right) \in \mathscr{E}_{\mathbf{R}}=\left\{\left(y_{1}, \ldots, y_{n}\right) \in\left(\boldsymbol{R}^{4}\right)^{n} \mid y_{i} \neq y_{j} \quad \text { if } i \neq j\right\} .
$$

The $\mathfrak{X}_{\mathrm{R}}$ transform covariantly under the inhomogeneous Euclidean group, $i$ and $i^{*}$ under complex conjugate representations, and have the following symmetry property:

$$
\mathfrak{t}_{\pi(\mathrm{R})}\left(x_{\pi^{-1}(1)}, \ldots, x_{\pi^{-1}(n)}\right)=\sigma(\pi, \mathrm{R}) \mathfrak{X}_{\mathrm{R}}\left(x_{1}, \ldots, x_{n}\right) .
$$

The (multi-) matrices $\boldsymbol{C}_{\mathrm{R}}$ only depend on the transformation properties of the fields concerned.

Osterwalder and Schrader have shown ([1]) that the $\mathfrak{S}_{R}$, and thus also the $\ddot{\mathfrak{X}}_{\mathrm{R}}$, define distributions on the space of those Schwartz functions on $\left(\boldsymbol{R}^{4}\right)^{n}$ that vanish with all their derivatives whenever two arguments coincide.

Proposition 4. The $\mathfrak{X}_{\mathrm{R}}$ can be extended to distributions on $\mathscr{S}\left(\left(\boldsymbol{R}^{4}\right)^{n}\right)$ satisfying covariance and (2.5), (2.6) in distribution sense.

These extensions will also be called the re-defined Schwinger functions and denoted $\mathfrak{X}_{\mathbf{R}}$.

Main Theorem. For a field theory as above, there exists a non-degenerate indefinite inner product, complex vector space $(\mathscr{K},\langle\cdot, \cdot\rangle)$ with a vacuum vector $\Omega$, a unitary representation $\mathrm{V}(\mathrm{A}, \mathrm{B}, a)$ of the covering group of the inhomogeneous Euclidean group and fields $\Psi^{(i)}, i \in \mathscr{I}$ such that

$\Omega$ is invariant under $\mathrm{V}(\mathrm{A}, \mathrm{B}, a)$ and cyclic w.r.t. the $\Psi^{(i)}$, $\Psi(i)$ transforms covariantly under V(A, B, a) (see (4.59)), 
the $\Psi^{(i)}$ commute totally (anti-commute for pairs of fermi fields) in distribution sense,

the vacuum expectation values of products of $\Psi^{(i)}$ 's are the re-defined Schwinger functions,

and $\Psi^{\left(i^{*}\right)}=\Psi^{(i) \dagger}$, the adjoint of $\Psi(i)$ w.r.t. $\langle\cdot, \cdot\rangle$.

Remark. The fact that $\Psi_{\alpha}^{(i)}(\mathrm{f})$ has an adjoint defined on all of $\mathscr{K}$ obviously implies that it is continuous in the weak topology defined by $\langle\cdot, \cdot\rangle$.

In fact, using Theorem III.5.1 of [14], it is easy to see that an operator on a non-degenerate inner product space has an everywhere defined adjoint (unique by non-degenerateness) if and only if it is weakly continuous.

\section{§3. The Free Dirac Field}

For convenience, let us fix the following representation of the $\gamma$-matrices:

$$
\gamma^{0}=\left(\begin{array}{ll}
0 & \mathbf{1} \\
\mathbf{1} & 0
\end{array}\right), \quad \gamma^{i}=\left(\begin{array}{cc}
0 & -\sigma_{i} \\
\sigma_{i} & 0
\end{array}\right) \quad i=1,2,3
$$

where $\sigma_{i}$ are the Pauli matrices. Throughout we let $\mathbb{1}$ denote a suitable unit matrix or operator.

Then

$$
\gamma^{0^{\alpha}}=\gamma^{0}, \quad \gamma^{i^{*}}=-\gamma^{i} \quad i=1,2,3 .
$$

The corresponding representation of $\operatorname{SL}(2, \boldsymbol{C})$ is

$$
\mathbf{S}(\mathrm{A})=\left(\begin{array}{cc}
\mathrm{A} & 0 \\
0 & \mathrm{~A}^{*-1}
\end{array}\right), \quad \mathrm{A} \in S L(2, \mathbb{C}) .
$$

We let $\Lambda$ denote the standard homomorphism of $S L(2, \mathbb{C})$ onto $\mathrm{L}_{+}^{\uparrow}$ (the restricted Lorentz group) given by:

$$
\begin{aligned}
(\Lambda(\mathrm{A}) x)_{\sim}=\mathrm{A}_{\sim} \mathrm{A}^{*}, x & =x^{0} \mathbb{1}+x^{i} \sigma_{i} \\
\text { for } & x \in \boldsymbol{R}^{4}\left(\text { or } \mathbb{C}^{4}\right), \mathrm{A} \in \operatorname{SL}(2, \mathbb{C}) .
\end{aligned}
$$

Analytic continuation of (3.3) and (3.4) give representations of $S L(2, \mathbb{C})$ $\times S L(2, \boldsymbol{C})$, the covering of the complex Lorentz group. For $\mathrm{A}, \mathrm{B} \in \operatorname{SL}(2, \mathbb{C})$, $z \in \mathbb{C}^{4}$ :

$$
\begin{aligned}
& \mathbf{S}(\mathrm{A}, \mathrm{B})=\left(\begin{array}{cc}
\mathrm{A} & 0 \\
0 & \mathrm{~B}^{\mathrm{T}^{-1}}
\end{array}\right) \\
& (\Lambda(\mathrm{A}, \mathrm{B}) z)_{\sim}=\mathrm{A} z \mathrm{~B}^{\mathrm{T}} .
\end{aligned}
$$


By restriction, (3.6) gives the following (universal covering) homomorphism of $S U(2) \times S U(2)$ onto the homogeneous Euclidean group in four dimensions

$$
(\mathrm{L}(\mathrm{A}, \mathrm{B}) x)^{\prime}=\Lambda(\mathrm{A}, \mathrm{B}) x^{\prime}
$$

where $\mathrm{A}, \mathrm{B} \in S U(2), x \in \boldsymbol{R}^{4}$ and $x^{\prime}$ is defined by (2.3).

(A) Re-defining the Schwinger Functions; the proof of Lemma 1.

The two-point Schwinger functions of the free Dirac field (defined like before (2.3), with $\mathrm{R}$ corresponding to $\psi \psi^{*}$ ) are (cf. [5])

$$
\varsigma^{0}(x, y)=(2 \pi)^{-4} \int\left(p^{2}+m^{2}\right)^{-1} \exp (i p(x-y))\left(i p^{E}+m\right) \gamma_{0}^{\mathrm{E}} \mathrm{d}^{4} p
$$

for $x \neq y$ in $\mathbb{R}^{4}$. Here $p x=\sum_{0}^{3} p_{a} x_{a}, \not^{\mathrm{E}}=\sum_{0}^{3} p_{a} \gamma_{a}^{\mathrm{E}}$ and the Euclidean $\gamma$-matrices are

$$
\gamma_{0}^{\mathrm{E}}=\gamma_{0}=\gamma^{0}, \gamma_{j}^{\mathrm{E}}=\mathrm{i} \gamma_{j}=-i \gamma^{j} \quad j=1,2,3 .
$$

Then

$$
\begin{aligned}
& \gamma_{a}^{\mathrm{E} *}=\gamma_{a}^{\mathrm{E}} \\
& \left\{\gamma_{a}^{\mathrm{E}}, \gamma_{b}^{\mathrm{E}}\right\}=2 \delta_{a b} \mathbb{1} \quad a, b=0, \ldots, 3 .
\end{aligned}
$$

$\mathfrak{S}^{0}$ is not hermitian, the reason why independent fields $\Psi^{(1)}, \Psi^{(2)}$ are introduced in [5]. The proof of our Lemma 1 is now immediate: If $\mathfrak{X}^{0}(x, y)$ $=\mathfrak{S}^{0}(x, y)\left(\mathbf{D} \gamma_{0}^{\mathrm{E}}\right)^{*}$ with $\mathbf{D}$ invertible, hermiticity of $\mathfrak{X}^{0}$ is equivalent to:

$$
\left\{\begin{array}{l}
\mathbb{D}^{*}=\mathbf{D} \\
\gamma_{a}^{\mathrm{E}} \mathbf{D}^{*}=-\mathbf{D} \gamma_{a}^{\mathrm{E}} \quad a=0, \ldots, 3,
\end{array}\right.
$$

and one solution is $\mathbf{D}=\gamma_{5}^{\mathrm{E}}=\gamma_{0}^{\mathrm{E}} \gamma_{1}^{\mathrm{E}} \gamma_{2}^{\mathrm{E}} \gamma_{3}^{\mathrm{E}}=\gamma_{5}^{\mathrm{E} *}=\left(\begin{array}{rr}1 & 0 \\ 0 & -\mathbb{1}\end{array}\right)$.

Uniqueness: $\quad \mathbf{D}=\mathbb{B} \gamma_{5}^{\mathrm{E}}$ is a solution of $(3.12,13)$ if and only if

$$
\left\{\begin{array}{l}
\gamma_{5}^{\mathrm{E}} \mathbf{B}^{*}=\mathbf{B} \gamma_{5}^{\mathrm{E}} \\
\gamma_{a}^{\mathrm{E}} \gamma_{5}^{\mathrm{E}} \mathbf{B}^{*}=-\mathbf{B} \gamma_{5}^{\mathrm{E}} \gamma_{a}^{\mathrm{E}}=\mathbf{B} \gamma_{a}^{\mathrm{E}} \gamma_{5}^{\mathrm{E}}
\end{array}\right.
$$

and hence $\left[\gamma_{a}^{\mathrm{E}}, \mathbf{B}\right]=0$, which by Schur's lemma implies $\mathbf{B}=\alpha \cdot \mathbb{1}, \alpha \in \mathbb{C}$. Either equation and invertibility of $\mathbb{D}$ gives $\alpha \in \mathbb{R} \backslash\{0\}$, and this proves Lemma 1 .

Q.E.D.

The two-point re-defined Schwinger functions are

$$
\mathfrak{X}^{0}(x, y)=(2 \pi)^{-4} \int\left(p^{2}+m^{2}\right)^{-1} \exp (i p(x-y))\left(i p^{\mathrm{E}}+m\right) \gamma_{5}^{\mathrm{E}} \mathrm{d}^{4} p
$$

which are extended to all $x, y \in \boldsymbol{R}^{4}$ as distributions on $\mathscr{S}\left(\left(\boldsymbol{R}^{4}\right)^{2}\right)$. 
The many point re-defined Schwinger functions are obtained from (3.14) by the following type of relations:

$$
\begin{aligned}
& \mathfrak{X}^{0}\left(x_{k}, \ldots, x_{1}, y_{1}, \ldots, y_{k}\right)=\sum_{\pi \in \mathscr{P}_{k}} \pm \mathfrak{X}^{0}\left(x_{1}, y_{\pi(1)}\right) \cdots \mathfrak{X}^{0}\left(x_{k}, y_{\pi(k)}\right) \\
& \mathfrak{X}^{0}\left(x_{k}, \ldots, x_{1}, y_{1}, \ldots, y_{\ell}\right)=0 \text { if } k \neq l .
\end{aligned}
$$

Here the arguments $x_{i}$ correspond to fields $\psi, y_{i}$ to $\psi^{*}$.

(B) Constructing the Euclidean theory; the proof of Proposition 1.

By continuing the covariance properties from the relativistic region we obtain for $\mathrm{A}, \mathrm{B} \in S U(2), a \in \mathbb{R}^{4}$ and distinct $x, y$ in $\mathbb{R}^{4}$ :

$$
\mathfrak{\subseteq}^{0}(\mathrm{~L}(\mathrm{~A}, \mathrm{~B}) x+a, \mathrm{~L}(\mathrm{~A}, \mathrm{~B}) y+a)=\mathbf{S}(\mathrm{A}, \mathrm{B}) \mathfrak{S}^{0}(x, y) \mathbf{S}(\mathrm{B}, \mathrm{A})^{\mathrm{T}} \text {. }
$$

Hence

$$
\begin{aligned}
\mathfrak{X}^{0}(\mathrm{~L}(\mathrm{~A}, \mathrm{~B}) x+a, \mathrm{~L}(\mathrm{~A}, \mathrm{~B}) y+a) & =\mathbf{S}(\mathrm{A}, \mathrm{B}) \mathfrak{K}^{0}(x, y) \gamma_{5}^{\mathrm{E}} \gamma_{0}^{\mathrm{E}} \mathrm{S}(\mathrm{B}, \mathrm{A})^{\mathrm{T}} \gamma_{0}^{\mathrm{E}} \gamma_{5}^{\mathrm{E}} \\
& =\mathbf{S}(\mathrm{A}, \mathrm{B}) \mathfrak{X}^{0}(x, y) \mathbf{S}(\mathrm{A}, \mathrm{B})^{*} .
\end{aligned}
$$

(3.16) remains true in distribution sense for all $x, y \in \mathbb{R}^{4}$.

We now start the construction of the "state space" of Proposition 1. Not unexpectedly it will be the (incomplete) anti-symmetric Fock space over a certain "one particle space".

Let $\mathrm{K}$ be the space $\mathbb{C}^{8} \otimes \mathscr{S}\left(\mathbb{R}^{4}\right)$, where $\mathscr{S}$ is the Schwartz space of rapidly decreasing $C^{\infty}$ functions.

If the pairs $\left[\mathrm{f}_{i}, \mathrm{~g}_{i}\right] \in \mathrm{K}$, where $\mathrm{f}_{i}, \mathrm{~g}_{i} \in \mathbb{C}^{4} \otimes \mathscr{S}\left(\mathbb{R}^{4}\right), i=1,2$, define the nondegenerate indefinite inner product

$$
\left\langle\left[f_{1}, g_{1}\right],\left[f_{2}, g_{2}\right]\right\rangle=\left|f_{1}, f_{2}\right|+\left|g_{1}, g_{2}\right|
$$

where

$$
\left|\mathrm{f}_{1}, \mathrm{f}_{2}\right|=-\int \mathrm{f}_{1}(x)^{*} \overline{\mathfrak{X}^{0}(x, y)} \mathrm{f}_{2}(y) d^{4} x d^{4} y
$$

and

$$
\mid \mathrm{g}_{1}, \mathrm{~g}_{2} /=\int \mathrm{g}_{1}(x)^{*} \mathfrak{X}^{0}(x, y) \mathrm{g}_{2}(y) d^{4} x d^{4} y
$$

The representation $\mathrm{v}(\mathrm{A}, \mathrm{B}, a)$ of the covering of inhomogeneous $S O(4)$, defined by

$$
\begin{aligned}
(\mathrm{v}(\mathrm{A}, \mathrm{B}, a)[\mathrm{f}, \mathrm{g}])(x)= & {\left[\mathrm{S}\left(\mathrm{A}^{-1}, \mathrm{~B}^{-1}\right)^{\mathrm{T}} \mathrm{f}\left(\mathrm{L}(\mathrm{A}, \mathrm{B})^{-1}(x-a)\right),\right.} \\
& \left.\mathrm{S}\left(\mathrm{A}^{-1}, \mathrm{~B}^{-1}\right)^{*} \mathrm{~g}\left(\mathrm{~L}(\mathrm{~A}, \mathrm{~B})^{-1}(x-a)\right)\right],
\end{aligned}
$$

is then unitary w.r.t. $\langle\cdot, \cdot\rangle$, by (3.16). 
Define $\mathscr{K}$ to be the algebraic direct sum of $\Lambda_{0}^{m}(\mathrm{~K})$ for $m=0,1, \ldots$, where $\Lambda_{0}^{m}(\mathrm{~K})$ is the algebraic $m$-th exterior product of $\mathrm{K}, \Lambda_{0}^{0}(\mathrm{~K})=\boldsymbol{C}$. I.e. $\mathscr{H}$ consists of all elements of the anti-symmetric Fock space over $\mathrm{K}$ with only a finite number of components non-zero.

The non-degenerate indefinite inner product $\langle\cdot, \cdot\rangle$ on $\mathscr{K}$ is, as usual, defined by sesqui-linear extension of

$$
\left\langle\xi_{1} \wedge \cdots \wedge \xi_{m}, \eta_{1} \wedge \cdots \wedge \eta_{n}\right\rangle=\delta_{m n} \operatorname{det}\left(\left\langle\xi_{i}, \eta_{j}\right\rangle\right)
$$

where $\xi_{i}, \eta_{i} \in \mathrm{K}$. The representation $\mathrm{V}$ in Proposition 1 is defined by linear extension of

$$
\mathrm{V}(\mathrm{A}, \mathrm{B}, a)\left(\xi_{1} \wedge \cdots \wedge \xi_{n}\right)=\left(\mathrm{v}(\mathrm{A}, \mathrm{B}, a) \xi_{1}\right) \wedge \cdots \wedge\left(\mathrm{v}(\mathrm{A}, \mathrm{B}, a) \xi_{n}\right),
$$

where $\mathrm{A}, \mathrm{B} \in S U(2), a \in \boldsymbol{R}^{4}, \xi_{i} \in \mathrm{K}$. It is obviously unitary w.r.t. $\langle\cdot, \cdot\rangle$ and it leaves the vector $\Omega=1 \in \Lambda_{0}^{0}(\mathrm{~K})$ invariant.

To complete the proof of Proposition 1 it only remains to construct the fields $\Psi$ and $\Psi^{\dagger}$ and verify their properties. For $\xi \in \mathrm{K}$, define the creation operator $\mathrm{B}^{\dagger}(\xi)$ as a linear operator on $\mathscr{K}$ satisfying

$$
\mathrm{B}^{\dagger}(\xi)\left(\xi_{1} \wedge \cdots \wedge \xi_{n}\right)=\xi \wedge \xi_{1} \wedge \cdots \wedge \xi_{n} .
$$

Its adjoint, the annihilation operator $\mathrm{B}(\xi)$, acts on $\mathscr{K}$ linearly and satisfies

$$
\mathrm{B}(\xi)\left(\xi_{1} \wedge \cdots \wedge \xi_{\mathrm{n}}\right)=\sum_{i=1}^{n}(-1)^{i+1}\left\langle\xi, \xi_{i}\right\rangle \xi_{1} \wedge \cdots \wedge \xi_{i-1} \wedge \xi_{i+1} \wedge \cdots \wedge \xi_{n} .
$$

$\mathrm{B}(\xi)$ obviously depends conjugate linearly on $\xi$.

As usual, for $\xi, \eta \in \mathrm{K}$ :

$$
\begin{aligned}
& \left\{\mathrm{B}(\xi), \mathrm{B}^{\dagger}(\eta)\right\}=\langle\xi, \eta\rangle \\
& \{\mathrm{B}(\xi), \mathrm{B}(\eta)\}=\left\{\mathrm{B}^{\dagger}(\xi), \mathrm{B}^{\dagger}(\eta)\right\}=0 .
\end{aligned}
$$

Obviously, for $\xi \in \mathrm{K}, \mathrm{A}, \mathrm{B} \in S U(2), a \in \boldsymbol{R}^{4}$ :

$$
\mathrm{V}(\mathrm{A}, \mathrm{B}, a) \mathrm{B}^{(\dagger)}(\xi) \mathrm{V}(\mathrm{A}, \mathrm{B}, a)^{-1}=\mathrm{B}^{(\dagger)}(\mathrm{v}(\mathrm{A}, \mathrm{B}, a) \xi) .
$$

We now define $\Psi^{\left({ }^{\dagger}\right)}(\mathrm{h}): \mathscr{K} \rightarrow \mathscr{K} \quad$ for $\quad \mathrm{h} \in \boldsymbol{C}^{4} \otimes \mathscr{S}\left(\boldsymbol{R}^{4}\right)$ by

$$
\begin{aligned}
& \Psi(\mathrm{h})=\mathrm{B}^{\dagger}([\mathrm{h}, 0])+\mathrm{B}([0, \overline{\mathrm{h}}]) \\
& \Psi^{\dagger}(\mathrm{h})=\mathrm{B}([\overline{\mathrm{h}}, 0])+\mathrm{B}^{\dagger}([0, \mathrm{~h}]) .
\end{aligned}
$$

Note that, unlike B, both $\Psi(\mathrm{h})$ and $\Psi^{\dagger}(\mathrm{h})$ depend linearly on $\mathrm{h}$ and $\Psi(\mathrm{h})^{\dagger}$ $=\Psi^{\dagger}(\overline{\mathrm{h}})$.

To prove that any vector in $\mathscr{K}$ can be written as a polynomial in $\Psi\left(\mathrm{f}_{i}\right)$ and 
$\Psi^{\dagger}\left(\mathrm{g}_{j}\right)$ acting on $\Omega$, i.e. that $\Omega$ is cyclic, we note that any vector can be written as a finite sum of vectors of the form $\left[\mathrm{f}_{1}, 0\right] \wedge \cdots \wedge\left[\mathrm{f}_{k}, 0\right] \wedge\left[0, \mathrm{~g}_{1}\right] \wedge \cdots \wedge\left[0, \mathrm{~g}_{l}\right]$. For each of these the result follows from the fact that it is the highest order term in $\Psi\left(\mathrm{f}_{1}\right) \ldots \Psi\left(\mathrm{f}_{k}\right) \Psi^{\dagger}\left(\mathrm{g}_{1}\right) \ldots \Psi^{\dagger}\left(\mathrm{g}_{\ell}\right) \Omega$, and by using induction on the order $k+l$.

To prove covariance, use (3.24) and (3.17) to obtain

$$
\mathrm{V}(\mathrm{A}, \mathrm{B}, a)^{-1} \Psi(\mathrm{h}) \mathrm{V}(\mathrm{A}, \mathrm{B}, a)=\Psi(\tilde{\mathrm{h}})
$$

where $\tilde{\mathrm{h}}(x)=\mathbf{S}(\mathrm{A}, \mathrm{B})^{\mathrm{T}} \mathrm{h}(\mathrm{L}(\mathrm{A}, \mathrm{B}) x+a)$. Hence

$$
\mathrm{V}(\mathrm{A}, \mathrm{B}, a)^{-1} \Psi(x) \mathrm{V}(\mathrm{A}, \mathrm{B}, a)=\mathrm{S}(\mathrm{A}, \mathrm{B}) \Psi\left(\mathrm{L}(\mathrm{A}, \mathrm{B})^{-1}(x-a)\right)
$$

and the adjoint relation:

$$
\mathrm{V}(\mathrm{A}, \mathrm{B}, a)^{-1} \Psi^{\dagger}(x) \mathrm{V}(\mathrm{A}, \mathrm{B}, a)=\overline{\mathrm{S}(\mathrm{A}, \mathrm{B})} \Psi^{\dagger}\left(\mathrm{L}(\mathrm{A}, \mathrm{B})^{-1}(x-a)\right)
$$

i.e. the desired covariance.

From $(3.22,23)$ we find

$$
\begin{gathered}
\left\{\Psi(\mathrm{f}), \Psi^{\dagger}(\mathrm{g})\right\}=\langle[\overline{\mathrm{g}}, 0],[\mathrm{f}, 0]\rangle+\langle[0, \overline{\mathrm{f}}],[0, \mathrm{~g}]\rangle \\
=|\overline{\mathrm{g}}, \mathrm{f} \backslash+| \overline{\mathrm{f}}, \mathrm{g} /=0 \\
\{\Psi(\mathrm{f}), \Psi(\mathrm{g})\}=\left\{\Psi^{\dagger}(\mathrm{f}), \Psi^{\dagger}(\mathrm{g})\right\}=0
\end{gathered}
$$

i.e. the fields anticommute totally.

For the vacuum expectation values, finally:

$$
\begin{aligned}
& \Psi(\mathrm{f}) \Psi^{\dagger}(\mathrm{g}) \Omega=\Psi(\mathrm{f})[0, \mathrm{~g}]=[\mathrm{f}, 0] \wedge[0, \mathrm{~g}]+\langle[0, \overline{\mathrm{f}}],[0, \mathrm{~g}]\rangle \Omega \\
& \left\langle\Omega, \Psi(\mathrm{f}) \Psi^{\dagger}(\mathrm{g}) \Omega\right\rangle=\langle[0, \overline{\mathrm{f}}],[0, \mathrm{~g}]\rangle=/ \overline{\mathrm{f}}, \mathrm{g} /=\mathfrak{X}^{0}(\mathrm{f}, \mathrm{g}) .
\end{aligned}
$$

Because of the general connection between Fock space and free fields it is easy to show that all re-defined Schwinger functions are obtained as vacuum expectation values of the fields $\Psi$ and $\Psi^{\dagger}$.

This completes the proof of Proposition 1.

Q.E.D.

(C) Making $\mathscr{K}$ a Hilbert space; the proof of Proposition 2.

Proposition 2 is proved by explicitly exhibiting the positive definite inner product majorizing $\langle\cdot, \cdot\rangle$ on $\mathscr{K}$ constructed above. This is sufficient, since $(\mathscr{K},\langle\cdot, \cdot\rangle)$ of Proposition 1 is unique up to unitary equivalence.

Let $(\cdot, \cdot)$ on $\mathrm{K} \times \mathrm{K}$ be defined by

$$
\begin{aligned}
\left(\left[\mathrm{f}_{1}, \mathrm{~g}_{1}\right],\left[\mathrm{f}_{2}, \mathrm{~g}_{2}\right]\right)= & \int \mathrm{f}_{1}(x)^{*} \delta_{m}(x-y) \mathrm{f}_{2}(y) d^{4} x d^{4} y \\
& +\int \mathrm{g}_{1}(x)^{*} \delta_{m}(x-y) \mathrm{g}_{2}(y) d^{4} x d^{4} y
\end{aligned}
$$


where (the distribution)

$$
\delta_{m}(x)=(2 \pi)^{-4} \int\left(p^{2}+m^{2}\right)^{-1 / 2} \exp (i p x) d^{4} p
$$

(cf. p. 284 in $[5]) . \quad(\cdot, \cdot)$ is positive definite.

Since $\mathrm{S}(\mathrm{A}, \mathrm{B})$ is a unitary matrix for $\mathrm{A}, \mathrm{B} \in \mathrm{SU}(2), \mathrm{v}(\mathrm{A}, \mathrm{B}, a)$ is unitary w.r.t. $(\cdot, \cdot)$ on $\mathrm{K}$.

It is easy to see that $(\cdot, \cdot)$ defines a decomposition majorant on $\mathrm{K}$, in the sense of Bognár [14]. I.e. there is an operator $\mathrm{J}$ on $\mathrm{K}$, with $\mathrm{J}^{2}=\mathbb{1}$, which is self-adjoint and unitary both w.r.t. $\langle\cdot, \cdot\rangle$ and w.r.t. $(\cdot, \cdot)$, such that

$$
\langle\cdot, \cdot\rangle=(\cdot, \mathrm{J} \cdot) \text {. }
$$

$\mathbf{J}$ can be expressed as

$$
(\mathrm{J}[\mathrm{f}, \mathrm{g}])(x)=\left[\int \overline{-j_{m}(x-y)} \mathrm{f}(y) d^{4} y, \int j_{m}(x-y) \mathrm{g}(y) d^{4} y\right]
$$

where (the distribution)

$$
j_{m}(x)=(2 \pi)^{-4} \int\left(p^{2}+m^{2}\right)^{-1 / 2} \exp (i p x)\left(i p^{\mathrm{E}}+m\right) \gamma_{5}^{\mathrm{E}} d^{4} p .
$$

Now we define $(\cdot, \cdot)$ on $\mathscr{K}$ by a definition like (3.18). This gives a positive definite inner product on $\mathscr{K}$ and the unitarity of $\mathrm{V}(\mathrm{A}, \mathrm{B}, a)$ w.r.t. it is clear. Extending $\mathrm{J}$ to $\mathscr{K}$, we see that $(\cdot, \cdot)$ on $\mathscr{K}$ defines a decomposition majorant of $\langle\cdot, \cdot\rangle$ on $\mathscr{K}$.

This proves Proposition 2.

Q.E.D.

Remark 1. Positive definite inner products on $\mathscr{K}$ which make $\mathrm{V}$ unitary and majorizing the indefinite inner product $\langle\cdot, \cdot\rangle$ are not uniquely defined even if we restrict our attention to those defined from a decomposition majorant on $\mathrm{K}$ (like in (3.18)).

In fact, if $(\cdot, \cdot)_{1}=\left\langle\cdot, \mathrm{J}_{1} \cdot\right\rangle$ is a positive definite inner product on $\mathrm{K}$ with $\mathbf{J}_{1}$ a fundamental symmetry, $\mathrm{v}$ is unitary w.r.t. $(\cdot, \cdot)_{1}$ if and only if $\mathrm{J}_{1} \mathrm{v}=\mathrm{vJ}_{1}$. This relation for $\mathrm{v}(\mathbb{1}, \mathbb{1}, a), a \in \boldsymbol{R}^{4}$, shows that $\mathrm{J}_{1}$ must act as multiplication with some $p$-dependent $(8 \times 8)$ matrix on the Fourier transform. If we let this matrix be

$$
\left(p^{2}+m^{2}\right)^{-1 / 2}\left(\begin{array}{cc}
\overline{-\left(i p^{\mathrm{E}}+m\right) \gamma_{5}^{\mathrm{E}}} & 0 \\
0 & \left(i p^{\mathrm{E}}+m\right) \gamma_{5}^{\mathrm{E}}
\end{array}\right) \mathrm{H}(p),
$$

the $\mathrm{J}$ of (3.35) corresponds to the matrix $\mathrm{H}(p)$ being $\mathbb{1}$. 
A detailed analysis, however, shows that any positive matrix $\mathrm{H}(p)$ such that the square of (3.38) is $\mathbb{1}$ and

$$
\mathrm{H}(p)\left(\begin{array}{cc}
\overline{\mathbf{S}(\mathrm{A}, \mathrm{B})} & 0 \\
0 & \mathbf{S}(\mathrm{A}, \mathrm{B})
\end{array}\right)=\left(\begin{array}{cc}
\overline{\mathbf{S}(\mathrm{A}, \mathrm{B})} & 0 \\
0 & \mathrm{~S}(\mathrm{~A}, \mathrm{~B})
\end{array}\right) \mathrm{H}\left(\mathrm{L}(\mathrm{A}, \mathrm{B})^{-1} p\right)
$$

will do.

Without giving the general form of such $\mathrm{H}$, we note that

$$
\mathrm{H}(p)=\left(\begin{array}{cc}
\cosh \varphi_{1}\left(p^{2}\right) \mathbb{1}+\sinh \varphi_{1}\left(p^{2}\right) \frac{p^{\mathrm{E}}}{|p|} & 0 \\
0 & \cosh \varphi_{2}\left(p^{2}\right) \mathbb{1}+\sinh \varphi_{2}\left(p^{2}\right) \frac{p^{\mathrm{E}}}{|p|}
\end{array}\right)
$$

is one, for any (continuous, say) real-valued functions $\varphi_{1}$ and $\varphi_{2}$ with $\varphi_{i}(0)=0$.

This shows that the positive definite inner product is not unique, as asserted.

Remark 2. Let $\mathrm{I}_{s}$ be space inversion on $\mathbb{R}^{4}$, i.e. the operation of changing sign of all components except the first one. Then

$$
\mathfrak{X}^{0}\left(I_{s} x, I_{s} y\right)=-\gamma_{0}^{\mathrm{E}} \mathfrak{X}^{0}(x, y) \gamma_{0}^{\mathrm{E}}
$$

for $x, y \in \mathbb{R}^{4}$, as is easily seen from (3.14).

We now show that the Euclidean Dirac theory is not space inversion invariant, in the following sense:

On $\mathscr{K}$ there is no unitary operator $\mathrm{V}\left(\mathbb{I}_{s}\right)$ such that

$$
\begin{gathered}
\mathrm{V}\left(\mathrm{I}_{s}\right) \mathrm{V}(\mathrm{A}, \mathrm{B}, a)=\mathrm{V}\left(\mathrm{B}^{\mathrm{T}^{-1}}, \mathrm{~A}^{\mathrm{T}^{-1}}, \mathrm{I}_{s} a\right) \mathrm{V}\left(\mathrm{I}_{s}\right) \\
\mathrm{V}\left(\mathrm{I}_{s}\right) \Omega=\Omega \\
\mathrm{V}\left(\mathrm{I}_{s}\right)^{-1} \Psi(x) \mathrm{V}\left(\mathrm{I}_{s}\right)=\mathrm{P}^{\top}\left(\mathrm{I}_{s} x\right)+\mathrm{Q}^{\top \dagger}\left(\mathrm{I}_{s} x\right) .
\end{gathered}
$$

Here $P$ and $Q$ are $4 \times 4$ matrices. (3.42) is the representation condition for the full Euclidean group.

Indeed, for unitary V (3.43), (3.44) and the adjoint of (3.44) imply, using (3.41),

$$
\mathfrak{X}^{0}(x, y)=-\mathbf{P} \gamma_{0}^{\mathrm{E}} \mathfrak{X}^{0}(x, y) \gamma_{0}^{\mathrm{E}} \mathrm{P}^{*}+\overline{\mathrm{Q}} \gamma_{0}^{\mathrm{E}} \mathfrak{X}^{0}(y, x) \gamma_{0}^{\mathrm{E}} \mathrm{Q}^{\mathrm{T}} .
$$

(3.42) and (3.44), however, give the following general form of $\mathrm{P}$ and $\mathrm{Q}$ :

$$
\mathrm{P}=\left(\begin{array}{cc}
0 & \alpha_{1} \cdot \mathbb{1} \\
\alpha_{2} \cdot \mathbb{1} & 0
\end{array}\right), \quad \mathrm{Q}=\left(\begin{array}{cc}
0 & \beta_{1} \sigma_{2} \\
\beta_{2} \sigma_{2} & 0
\end{array}\right)
$$

with complex numbers $\alpha_{1,2}, \beta_{1,2}$ and $\sigma_{2}$ the second Pauli matrix. A simple 
computation using the expression (3.14) for $\mathfrak{X}^{0}(x, y)$ shows that (3.45) can not be satisfied for all $x, y \in \boldsymbol{R}^{4}$.

\section{§4. A Wightman Field Theory with Space Inversion Invariance}

Let $\mathrm{U}(\mathrm{A}, a), \mathrm{U}\left(\mathrm{I}_{s}\right)$ for $\mathrm{A} \in \mathrm{SL}(2, \boldsymbol{C}), a \in \boldsymbol{R}^{4}$ be the unitary representation of (the covering of) the orthochronous Poincare group on the relativistic state space. The fields $\psi_{y}$, transform as

$$
\begin{gathered}
\mathrm{U}(\mathrm{A}, a)^{-1} \psi_{\gamma}(x) \mathrm{U}(\mathrm{A}, a)=\mathbf{S}_{\gamma \gamma^{\prime}}(\mathrm{A}) \psi_{\gamma^{\prime}}\left(\Lambda(\mathrm{A})^{-1}(x-a)\right) \\
\mathrm{U}\left(\mathrm{I}_{s}\right)^{-1} \psi_{\gamma}(x) \mathrm{U}\left(\mathrm{I}_{s}\right)=\mathbf{P}_{\gamma \gamma^{\prime}} \psi_{\gamma^{\prime}}\left(\mathrm{I}_{s} x\right)
\end{gathered}
$$

where $\Lambda(\mathrm{A})$ is as in (3.4), for $\mathrm{A} \in S L(2, \boldsymbol{C})$. The matrices $\mathbf{S}(\mathrm{A}), \mathbf{P}$ give a representation (assumed to be continuous) of the covering group of the orthochronous Lorentz group, i.e.

$$
\mathbf{P S}(\mathrm{A})=\mathbf{S}\left(\mathrm{A}^{*-1}\right) \mathbf{P},
$$

but we do not assume that $\mathbf{P}^{2}=\mathbf{1}$. Further, by Section 2,

$$
\psi_{\gamma}(x)^{*}=\mathbf{D}_{\gamma \gamma^{\prime}} \psi_{\gamma^{\prime}}(x)
$$

for some matrix $\mathbf{D}$. It obviously satisfies $\overline{\mathbf{D}} \mathbf{D}=\mathbf{1}$.

(A) Choosing linear basis for the fields; the proof of Lemma 2.

Since the covering group of the orthochronous Lorentz group has an infinite number of components, its complexification does not have a compact real form and not all its finite-dimensional representations are completely reducible. In our case, however, the matrix $\mathbf{P}^{2}$ is equivalent to a unitary one and "the Weyl unitarity trick" can be used to prove that our representation $\mathbf{S}(\mathrm{A}), \mathbf{P}$ is completely reducible.

To prove that $\mathbf{P}^{2}$ is equivalent to a unitary, we show that all its eigenvalues are semi-simple and of absolute value 1 . Let $\psi$ be a field (i.e. a linear combination of the $\psi_{\gamma}$ ) such that

$$
\mathrm{U}\left(\mathrm{I}_{\mathrm{s}}\right)^{-2} \psi(x) \mathrm{U}\left(\mathrm{I}_{\mathrm{s}}\right)^{2}=\lambda \psi(x) .
$$

The vacuum vector $\omega$ satisfies $\mathrm{U}\left(\mathrm{I}_{s}\right) \omega=\omega$ and there exists a test function $\mathrm{f}$ such that $\xi=\psi(f) \omega \neq 0$, by the Reeh-Schlieder theorem. (4.5) then gives $U\left(I_{s}\right)^{-2} \xi$ $=\lambda \xi$ and hence $|\lambda|=1$. If the eigenvalue $\lambda$ of $\mathbf{P}^{2}$ were not semisimple, we could for some such $\psi$ find a field $\psi^{\prime}$ with 


$$
\mathrm{U}\left(\mathrm{I}_{s}\right)^{-2} \psi^{\prime}(x) \mathrm{U}\left(\mathrm{I}_{s}\right)^{2}=\psi(x)+\lambda \psi^{\prime}(x) .
$$

This would, however, imply that $\mathrm{U}\left(\mathrm{I}_{s}\right)^{-2} \eta=\xi+\lambda \eta$, for $\eta=\psi^{\prime}(\mathrm{f}) \omega$. Since $\mathrm{U}\left(\mathrm{I}_{s}\right)$ is unitary we have a contradiction, i.e. the eigenvalue $\lambda$ is semisimple and $\mathbf{P}^{2}$ equivalent to a unitary. (In this argument we obviously have to assume that $\mathrm{U}\left(\mathrm{I}_{s}\right)$ is unitary w.r.t. a positive definite inner product.)

$\mathrm{S}(\mathrm{A})$ gives a finite-dimensional, continuous representation of $S L(2, \mathbb{C})$ and can be analytically continued to $\mathrm{S}(\mathrm{A}, \mathrm{B})$, giving a representation of $S L(2, \mathbb{C})$ $\times S L(2, \boldsymbol{C})$. The relation continuing $(4.3)$ is

$$
\mathbf{P S}(A, B)=\mathbf{S}\left(B^{T^{-1}}, A^{T^{-1}}\right) \mathbf{P}
$$

for $\mathrm{A}, \mathrm{B} \in S L(2, \boldsymbol{C})$.

To prove Lemma 2, we consider the finite-dimensional vector space $\mathscr{V}$ which has basis vectors $\mathrm{e}_{\gamma}$, where the index $\gamma$ has the same range as for the fields $\psi_{\gamma}$. On $\mathscr{\gamma}^{\mathrm{S}} \mathrm{S}(\mathrm{A}, \mathrm{B})$ and $\mathbf{P}$ define a representation, $\mathrm{t}(\mathrm{A}, \mathrm{B}), \pi$, of the covering group of the full complex Lorentz group:

$$
\begin{gathered}
\mathrm{t}(\mathrm{A}, \mathrm{B})^{-1} \mathrm{e}_{\gamma^{\prime}}=\mathrm{S}_{\gamma \gamma^{\prime}}(\mathrm{A}, \mathrm{B}) \mathrm{e}_{\gamma^{\prime}} \\
\pi^{-1} \mathrm{e}_{\gamma}=\mathrm{P}_{\gamma \gamma^{\prime}} \mathrm{e}_{\gamma^{\prime}}
\end{gathered}
$$

for $\mathrm{A}, \mathrm{B} \in S L(2, \boldsymbol{C})$. For the restriction to the real Lorentz group we denote $\mathrm{t}(\mathrm{A})=\mathrm{t}(\mathrm{A}, \overline{\mathrm{A}}), \mathrm{A} \in S L(2, \boldsymbol{C})$. We also introduce an involution, *, on $\mathscr{V}:$

$$
* \mathrm{e}_{\gamma}=\mathbf{D}_{\gamma \gamma^{\prime}} \mathrm{e}_{\gamma^{\prime}} \text {. }
$$

It then follows from the unitarity of $U$ and $(4.1,2,4,7)$ :

$$
* \mathrm{t}(\mathrm{A})=\mathrm{t}(\mathrm{A}) * \text { and } \quad * \pi=\pi * .
$$

If we find a basis $\left\{\mathrm{c}_{\alpha_{i}}^{(i)}\right\}$ for $\mathscr{V}$, such that the $\left\{\mathrm{e}_{\alpha_{i}}^{(i)}\right\}$ for fixed $i$ transform irreducibly under $\mathrm{t}, \pi$ and also $* \mathrm{e}_{\sigma_{i}}^{(i)}=\mathrm{e}_{\alpha_{i}}^{(i *)}$ for some $i^{*}$, Lemma 2 will follow.

Since the matrix $\mathbf{P}^{2}$ is equivalent to a unitary, there exists a positive definite inner product on $\mathscr{V}$, which makes $\pi^{2}$ unitary. Let it be $(\cdot, \cdot)_{0}$ and define

$$
(\cdot, \cdot)=\sum_{i=0}^{1} \int\left(\pi^{i} \mathrm{t}(\mathrm{A}, \mathrm{B}) \cdot, \pi^{i} \mathrm{t}(\mathrm{A}, \mathrm{B}) \cdot\right)_{0} \mathrm{~d} \mu(\mathrm{A}, \mathrm{B})
$$

on $\mathscr{V} \times \mathscr{V}$. Here the integral is over $S U(2) \times S U(2)$ and $\mu$ is Haar measure on that group. It is then easy to see that $\mathrm{t}(\mathrm{A}, \mathrm{B})$ for $\mathrm{A}, \mathrm{B} \in S U(2)$ and $\pi$ are unitary w.r.t. $(\cdot, \cdot)$. This shows (using the analyticity of the $\mathbf{S}(\mathrm{A}, \mathrm{B})$ ) that $\mathscr{V}$ decomposes completely:

$$
\mathscr{V}=\sum_{v} \oplus \mathscr{\mathscr { N }}(v)
$$


where $v$ ranges over the (equivalence classes of) irreducible representations of the covering of the orthochronous Lorentz group and each $\mathscr{V}^{(v)}=\mathscr{M}^{(v)}$ $\otimes \mathscr{N}^{(v)} . \mathscr{H}^{(v)}$ is a standard representation space for the representation $v$ and $\mathscr{N}^{(v)}$ is a vector space with dimension the multiplicity of $v$ in $\mathrm{t}, \pi$. $\mathrm{t}(\mathrm{A})$ and $\pi$ act on $\mathscr{V}^{(v)}$ as

$$
\begin{aligned}
& \left.\mathrm{t}(\mathrm{A})\right|_{\mathscr{r}(v)}=\mathrm{t}^{(v)}(\mathrm{A}) \otimes \mathbb{1} \\
& \pi \operatorname{lor}(v)=\pi^{(v)} \otimes \mathbb{1} .
\end{aligned}
$$

Since $*$ is conjugate linear, (4.11) implies that $* \mathscr{V}^{(v)}=\mathscr{V}^{\left(v^{*}\right)}$, where $v^{*}$ is the representation complex conjugate to $v$.

If $v \neq v^{*}$ and $\left\{\mathrm{e}_{\alpha_{2}}^{(i)}\right\}$ is a basis for $\mathscr{V}^{(v)}$ which decomposes it into irreducible subspaces, the same will be true of $\left\{\mathrm{e}_{\alpha_{i}}^{(i *)}\right\}=\left\{* \mathrm{e}_{\alpha_{i}}^{(i)}\right\}$ in $\mathscr{V}^{\left(v^{+}\right)}$. So, in this case Lemma 2 holds.

If $v=v^{*}$ there exists a conjugate linear mapping, $\mathrm{q}^{(v)}$, on $\mathscr{M}^{(v)}$ which commutes with $\mathfrak{t}^{(v)}(\mathrm{A})(\mathrm{A} \in S L(2, C))$ and $\pi^{(v)}$ and such that $\mathrm{q}^{(v)^{2}}= \pm \mathbb{1}$ (the sign depending on whether $t^{(v)}(A), \pi^{(v)}$ is equivalent to a real representation or not). Let $\kappa$ be an involution on $\mathscr{N}^{(v)}$. Then the linear $*\left(\mathrm{q}^{(v)} \otimes \kappa\right)$ commutes with all $\mathrm{t}(\mathrm{A})$ and $\pi$ on $\mathscr{V}^{(v)}$ and therefore it is $\mathbb{1} \otimes \mathrm{F}$ on $\mathscr{V}^{(v)}$ for some linear $\mathrm{F}$. This gives

$$
*=\mathrm{q}^{(v)} \otimes \mathrm{q}^{\prime(v)} \quad \text { on } \quad \mathscr{V}^{(v)}
$$

with conjugate linear $\mathrm{q}^{(v)}, \mathrm{q}^{\prime(v)}$ and $\mathrm{q}^{(v)^{2}}= \pm 1, \mathrm{q}^{\prime(v)^{2}}= \pm \mathbb{1}$, the same sign for both.

If $\mathrm{q}^{(v)^{2}}=+1$ we can choose "real" bases of $\mathscr{M}^{(v)}$ and $\mathscr{N}^{(v)}$ and choose our $\mathrm{e}_{\alpha_{i}}^{(i)}$ as tensor products of them.

If $\mathrm{q}^{(v)^{2}}=-1$ it is not hard to prove (by induction) that there is a basis $\left\{\mathrm{g}^{(i)}\right\}$ of $\mathscr{N}^{(v)}$ such that for $i$ odd: $\mathrm{q}^{\prime(v)} \mathrm{g}^{(i)}=\mathrm{g}^{(i+1)}$. Taking the tensor product of this basis with any basis of $\mathscr{M}^{(v)}$ gives us a basis for $\mathscr{V}^{(v)}$ with the desired properties (with indices $i$ in $\mathfrak{f}^{*}$ ).

This, at last, proves Lemma 2.

Q.E.D.

Remark. If $v$ is equivalent to a real representation, the construction above gives a basis for the corresponding fields which is hermitian. This does not mean that we have to choose them like that. For physical reasons, or to have as few hermitian fermion fields as possible, we may prefer to use non-hermitian fields in our basis. 
(B) Redefining the Schwinger functions; the proof of Proposition 3.

In the basis given by Lemma 2 , the transformation properties of the fields are:

$$
\begin{aligned}
& \mathrm{U}(\mathrm{A}, a)^{-1} \psi^{(i)}(x) \mathrm{U}(\mathrm{A}, a)=\mathbb{S}^{(i)}(\mathrm{A}) \psi^{(i)}\left(\Lambda(\mathrm{A})^{-1}(x-a)\right) \\
& \mathrm{U}\left(\mathrm{I}_{s}\right)^{-1} \psi^{(i)}(x) \mathrm{U}\left(\mathrm{I}_{s}\right)=\mathbb{P}^{(i)} \psi^{(i)}\left(\mathrm{I}_{s} x\right)
\end{aligned}
$$

where summation over $\alpha$-indices is implied on the right hand side (but not over $i$ ). The continuation of $\mathbf{S}^{(i)}$ from $S L(2, \mathbb{C})$ to its complexification $S L(2, \mathbb{C}) \times$ $S L(2, \mathbb{C})$ will be denoted $\mathrm{S}^{(i)}(\mathrm{A}, \mathrm{B})$. Then, as in (4.7),

$$
\mathbb{P}^{(i)} \mathbf{S}^{(i)}(\mathrm{A}, \mathrm{B})=\mathbf{S}^{(i)}\left(\mathrm{B}^{\mathrm{T}^{-1}}, \mathrm{~A}^{\mathrm{T}^{-1}}\right) \mathbb{P}^{(i)}
$$

for $\mathrm{A}, \mathrm{B} \in S L(2, C)$. Since the representation " $i$ " is irreducible,

$$
\mathbf{P}^{(i)^{2}}=\mathrm{c}^{(i)} \mathbb{1} \text { and } \mathbf{S}^{(i)}(-\mathbb{1},-1)= \pm \mathbb{1}
$$

for some $\mathrm{c}^{(i)} \in \mathbb{C}$ with $\left|\mathrm{c}^{(i)}\right|=1$. The sign in the second relation of (4.20) depends on whether " $i$ " describes bosons or fermions.

The unitarity of $U$ and $(4.17,18)$ imply

$$
\mathbf{S}^{\left(i^{*}\right)}(\mathrm{A})=\overline{\mathbf{S}^{(i)}(\mathrm{A})}, \mathbb{P}^{\left(i^{*}\right)}=\overline{\mathbb{P}^{(i)}}
$$

and hence

$$
\mathrm{c}^{\left(i^{*}\right)}=\overline{\mathrm{c}^{(i)}} .
$$

In particular, for $i \in \mathrm{n}, \mathbf{S}^{(i)}(\mathrm{A}), \mathbb{P}^{(i)}$ and $\mathrm{c}^{(i)}$ are real. Using the condition (mentioned in $(A)$ above) for a representation to be equivalent to a real one and known properties of representations of $S L(2, \mathbb{C})$, it is not hard to prove that for " $i$ " indexing a hermitian field, $\mathrm{c}^{(i)}=1$ for boson and $\mathrm{c}^{(i)}=-1$ for fermion index " $i$ ".

For the continued representations, (4.21) gives

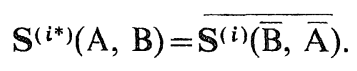

If $\mathrm{R}=i_{1} \cdots i_{n}$ is a sequence of indices in (our extended) $\mathscr{I}, \mathbb{S}_{\mathbf{R}}(\mathrm{A}, \mathrm{B})$ will denote the tensor product of $\mathbf{S}^{\left(i_{1}\right)}(\mathrm{A}, \mathrm{B}) \cdots \mathbf{S}^{\left(i_{n}\right)}(\mathrm{A}, \mathrm{B}) . \mathbf{S}_{\mathrm{R}_{\mathfrak{1}}}$ will be the analogue with the unit matrix instead of $\mathbf{S}^{\left(i_{\ell}\right)}(\mathrm{A}, \mathrm{B})$ if $i_{\ell} \notin \mathfrak{f} . \quad \mathbb{P}_{\mathbf{R}}, \mathbb{P}_{\mathbf{R} \cap \mathfrak{m}}, \mathbf{S}_{\mathbf{R} \backslash \mathfrak{\ell}^{*}}$ etc. are defined similarly.

The covariance properties of the Wightman functions can then be written

$$
\begin{gathered}
\mathfrak{W}_{\mathrm{R}}\left(\Lambda(\mathrm{A}, \mathrm{B}) z_{1}+a, \ldots, \Lambda(\mathrm{A}, \mathrm{B}) z_{n}+a\right)=\mathbf{S}_{\mathrm{R}}(\mathrm{A}, \mathrm{B}) \mathfrak{M}_{\mathrm{R}}\left(z_{1}, \ldots, z_{n}\right) \\
\mathfrak{D}_{\mathrm{R}}\left(\mathrm{I}_{s} z_{1}, \ldots, \mathrm{I}_{s} z_{n}\right)=\mathbb{P}_{\mathrm{R}} \mathfrak{B}_{\mathrm{R}}\left(z_{1}, \ldots, z_{n}\right)
\end{gathered}
$$


for $\mathrm{A}, \mathrm{B} \in S L(2, \boldsymbol{C}), a \in \boldsymbol{C}^{4}$ and $\left(z_{1}, \ldots, z_{n}\right)$ in the domain of definition of $\mathfrak{W}_{\mathrm{R}}$.

To prove Proposition 3, we observe that analytic continuation (by "the Edge of the Wedge theorem"') of the hermiticity conditions on the vacuum expectation values gives

$$
\mathfrak{W}_{\mathrm{R}}\left(z_{1}, \ldots, z_{n}\right)=\overline{\mathfrak{W}_{\mathrm{R}^{*}}\left(\bar{z}_{n}, \ldots, \bar{z}_{1}\right)} .
$$

Using $(4.24,25)$ we find

$$
\begin{aligned}
\mathfrak{W}_{\mathrm{R}}\left(z_{1}, \ldots, z_{n}\right) & =\overline{\mathbf{S}_{\mathrm{R}^{*}}(\mathbf{1},-\mathbf{1}) \mathfrak{Q}_{\mathrm{R}^{*}}\left(-\bar{z}_{n}, \ldots,-\bar{z}_{1}\right)} \\
& =\overline{\mathbf{S}_{\mathrm{R}^{*}}(\mathbf{1},-\mathbf{1}) \mathbf{P}_{\mathrm{R}^{*}} \mathfrak{W}_{\mathrm{R}^{*}}\left(-\mathrm{I}_{s} \bar{z}_{n}, \ldots,-\mathrm{I}_{s} \bar{z}_{1}\right)} .
\end{aligned}
$$

For the Schwinger functions this means

$$
\mathfrak{S}_{\mathbf{R}}\left(x_{1}, \ldots, x_{n}\right)=\overline{\mathbf{S}_{\mathrm{R}^{*}}(\mathbf{1},-\mathbf{1}) \mathbf{P}_{\mathrm{R}^{*}} \mathfrak{S}_{\mathrm{R}^{*}}\left(x_{n}, \ldots, x_{1}\right)}
$$

for $\left(x_{1}, \ldots, x_{n}\right) \in \mathscr{E}_{\mathrm{R}}$. Using (4.28) we will construct $\boldsymbol{C}_{\mathrm{R}}$ of Proposition 3.

Let $i \in \mathfrak{n}$. Since it is (by our extension of $\mathscr{I}$ ) a boson index, we have $c^{(i)}=1, \mathbf{S}^{(i)}(-\mathbf{1},-\mathbf{1})=\mathbf{1}$ and with

$$
\mathbf{C}^{(i)}=\frac{1+i}{2}\left(\mathbf{1}-i \mathbf{S}^{(i)}(\mathbf{1},-\mathbf{1}) \mathbf{P}^{(i)}\right)
$$

we find $\overline{\mathbf{C}^{(i)}-1} \mathbf{C}^{(i)}=\mathbf{S}^{(i)}(\mathbf{1},-\mathbf{1}) \mathbf{P}^{(i)}$ (e.g. by noting that $\left.\overline{\mathbf{C}^{(i)}}-1=\mathbf{C}^{(i)}\right)$.

Now, by $(4.23,21,19,20)$,

$$
\begin{aligned}
& \overline{\mathbf{S}_{\mathbf{R}^{*} \cap f}(1,-1) \mathbf{P}_{\mathbf{R}^{*} \cap \mathfrak{f}}}=\mathbf{S}_{\mathbf{R}_{\cap *}}(-1,1) \mathbf{P}_{\mathbf{R} \cap f^{*}} \\
& =\mathbf{P}_{\mathrm{R} \cap \mathfrak{t}^{*}}{ }^{-1} \mathbf{S}_{\mathrm{R} \cap \mathfrak{f}^{*}}(\mathbf{1},-\mathbf{1})^{-1} \prod_{\mathrm{R} \cap \mathrm{f}^{*}} \mathrm{c}^{(i)} .
\end{aligned}
$$

But using (4.25) twice we find that if $\mathfrak{M}_{\mathrm{R}}$ does not vanish, then $\prod_{\mathbb{R}} \mathrm{c}^{(i)}=1$. Hence, in this case,

$$
\prod_{\mathrm{R}_{n} \mathrm{f}^{*}} \mathrm{c}^{(i)}=\prod_{\mathrm{R}^{\prime} \backslash \mathrm{f}^{*}} \mathrm{C}^{(i)-1}=\prod_{\mathrm{R}^{*} \cap \mathrm{f}^{*}} \overline{\mathrm{c}^{(i)}-1}
$$

where we used (4.22) and $\mathrm{c}^{(i)}=1$ for $i \in \mathrm{n}$. If we define $z^{-1 / 2}$ so that $\arg z^{-1 / 2}$ $\in[0, \pi)$ for $z \in \boldsymbol{C} \backslash\{0\}$ (for instance),

$$
\left(\prod_{\mathrm{R} \cap f^{*}} \mathrm{c}^{(i)}\right)^{-1 / 2}=\overline{\left(\prod_{\mathrm{R}^{*} \cap \mathrm{f}^{*}} \mathrm{c}^{(i)}\right)^{-1 / 2}-1}
$$

if $\mathfrak{W}_{\mathrm{R}}$ does not vanish.

We now set

$$
\boldsymbol{C}_{\mathrm{R}}=\prod_{\mathrm{R} \cap \mathrm{n}} \mathbf{C}^{(i)}\left(\prod_{\mathrm{R} \cap \mathbb{E}^{*}} \mathrm{c}^{(i)}\right)^{-1 / 2} \mathbf{S}_{\mathrm{R} \cap *^{*}}(\mathbf{1},-\mathbf{1}) \mathbf{P}_{\mathrm{R} \cap t^{*}}
$$

and find that (4.28) shows that (2.5) is satisfied. 
The analyticity properties of $\mathfrak{\mathfrak { X }}_{\mathrm{R}}$ follow from the corresponding well-known properties of $\widetilde{\Xi}_{R}$.

The $\mathfrak{S}_{\mathbf{R}}$ transform covariantly under (the covering of) the inhomogeneous Euclidean group,

$$
\mathfrak{\Xi}_{\mathrm{R}}\left(\mathrm{L}(\mathrm{A}, \mathrm{B}) x_{1}+a, \ldots, \mathrm{L}(\mathrm{A}, \mathrm{B}) x_{n}+a\right)=\mathrm{S}_{\mathrm{R}}(\mathrm{A}, \mathrm{B}) \Im_{\mathrm{R}}\left(x_{1}, \ldots, x_{n}\right)
$$

for $\mathrm{A}, \mathrm{B} \in S U(2), a \in \boldsymbol{R}^{4}$ and $\left(x_{1}, \ldots, x_{n}\right) \in \mathscr{E}_{\mathrm{R}}$. Hence

$$
\mathfrak{X}_{\mathrm{R}}\left(\mathrm{L}(\mathrm{A}, \mathrm{B}) x_{1}+a, \ldots, \mathrm{L}(\mathrm{A}, \mathrm{B}) x_{n}+a\right)=\mathrm{S}_{\mathrm{R}}^{\prime}(\mathrm{A}, \mathrm{B}) \mathfrak{X}_{\mathrm{R}}\left(x_{1}, \ldots, x_{n}\right)
$$

where the $\mathbf{S}^{\prime(i)}(\mathrm{A}, \mathrm{B})$ for $\mathrm{A}, \mathrm{B} \in S U(2)$ are as follows.

For $i \in \mathfrak{f}$,

$$
\mathbf{S}^{\prime(i)}(\mathrm{A}, \mathrm{B})=\mathbf{S}^{(i)}(\mathrm{A}, \mathrm{B})
$$

For $i \in \mathfrak{f}^{*}$,

$$
\begin{aligned}
& \mathbf{S}^{\prime(i)}(\mathrm{A}, \mathrm{B})=\mathbf{S}^{(i)}(\mathbf{1},-\mathbf{1}) \mathbf{P}^{(i)} \mathbf{S}^{(i)}(\mathrm{A}, \mathrm{B}) \mathbf{P}^{(i)^{-1}} \mathbf{S}^{(i)}(\mathbb{1},-\mathbb{1})^{-1} \\
& =\mathbf{S}^{(i)}(\mathbb{1},-\mathbf{1}) \mathbf{S}^{(i)}\left(\mathrm{B}^{\mathrm{T}^{-1}}, \mathrm{~A}^{\mathrm{T}^{-1}}\right) \mathbf{S}^{(i)}(\mathbb{1},-\mathbb{1})^{-1}=\mathbf{S}^{(i)}(\overline{\mathrm{B}}, \overline{\mathrm{A}}) \\
& =\overline{\mathbf{S}^{(i)}(\mathrm{A}, \overline{\mathrm{B}})}=\overline{\mathbf{S}^{\prime\left(i^{*}\right)}(\mathrm{A}, \mathrm{B})}
\end{aligned}
$$

by (4.19), the unitarity of A, B and (4.23).

For $i \in \mathfrak{n}$ :

$$
\mathbf{S}^{(i)}(\mathrm{A}, \mathrm{B})=\mathbf{C}^{(i)} \mathbf{S}^{(i)}(\mathrm{A}, \mathrm{B}) \mathbf{C}^{(i)^{-1}}
$$

and from $\overline{\mathbf{C}^{(i)}-1} \mathbf{C}^{(i)}=\mathbf{S}^{(i)}(\mathbf{1},-\mathbf{1}) \mathbf{P}^{(i)}$ we find

$$
\mathbf{S}^{\prime(i)}(\mathrm{A}, \mathrm{B})=\overline{\mathbf{S}^{\prime(i)}(\mathrm{A}, \mathrm{B})} \text { for } i \in \boldsymbol{\pi} \text {. }
$$

Similarly,

$$
\mathfrak{\mathfrak { Z }}_{\mathrm{R}}\left(\mathrm{I}_{s} x_{1}, \ldots, \mathrm{I}_{s} x_{n}\right)=\mathbf{P}_{\mathrm{R}}^{\prime} \mathfrak{X}_{\mathrm{R}}\left(x_{1}, \ldots, x_{n}\right)
$$

where

$$
\begin{gathered}
\mathbf{P}^{\prime(i)}=\mathbf{P}^{(i)} \quad \text { if } \quad i \in \mathfrak{f} \\
\mathbf{P}^{\prime(i)}=\mathbf{S}^{(i)}(-\mathbf{1},-\mathbf{1}) \overline{\mathbf{P}^{\left(i^{*}\right)}} \quad \text { if } \quad i \in \mathfrak{f}^{*} \\
\mathbf{P}^{\prime(i)}=\mathbf{C}^{(i)} \mathbf{P}^{(i)} \mathbf{C}^{(i)^{-1}}=\overline{\mathbf{P}^{\prime(i)}} \quad \text { if } \quad i \in \mathfrak{n} .
\end{gathered}
$$

Since $\mathbf{C}_{\pi(\mathrm{R})}=\mathbf{C}_{\mathrm{R}}$, (2.6) follows from the corresponding result for $\mathfrak{S}_{\mathrm{R}}$.

This concludes the proof of Proposition 3.

Q.E.D.

Remark 1. From (4.33) we see that if we had restricted ourselves to the case

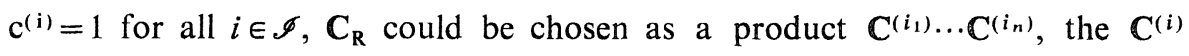
depending only on $i$ and being $\mathbb{1}$ if $i \in \mathfrak{f}$. 
For free fields it is always possible to choose $\mathrm{c}^{(i)}=1$ (the number operator can be used to modify $U\left(I_{s}\right)$ ), and in the Dirac case we could avoid extra phase factors in the redefinition of the Schwinger functions.

Remark 2. The Euclidean theory will in general not be space inversion invariant, because of the factor $\mathbf{S}^{(i)}(-\mathbf{1},-\mathbf{1})$ in $(4.42)$.

(C) Extending to all Euclidean points; the proof of Proposition 4.

For $i \in \mathscr{I}$, let $\mathscr{S}_{i}$ be the space of test functions with values in (the dual of) the representation space of $\mathbf{S}^{(i)}$, i.e. an element of $\mathscr{S}_{i}$ is of the form $\left(\mathrm{f}_{\alpha_{i}}\right)$ with each $\mathrm{f}_{\alpha_{i}} \in \mathscr{S}\left(\boldsymbol{R}^{4}\right)$, the Schwartz space.

For a sequence $\mathrm{R}=i_{1} \cdots i_{n}$ of indices from $\mathscr{I}_{i}$ let $\mathscr{S}_{\mathrm{R}}=\mathscr{S}_{i_{1}} \otimes \cdots \otimes \mathscr{S}_{i_{n}}$, the completed tensor product. Its elements will have components $\mathrm{f}_{\alpha_{i_{1}} \cdots \alpha_{i_{n}}} \in$ $\mathscr{S}\left(\left(\boldsymbol{R}^{4}\right)^{n}\right)$.

With $\pi(\mathbf{R})$ as in Section 2, define

$$
\mathscr{S}_{[\mathrm{R}]}=\bigoplus_{\pi \in \mathscr{P}_{n}}^{\prime}\left(\mathscr{S}_{\pi(\mathrm{R})} \oplus \mathscr{S}_{(\pi(\mathrm{R}))^{*}}\right)
$$

where $\oplus^{\prime}$ means that we do not repeat identical terms. $\mathscr{S}_{[\mathrm{R}]}$ is a direct sum of a finite number of copies of $\mathscr{S}\left(\left(\boldsymbol{R}^{4}\right)^{n}\right)$ and this defines a natural Fréchet space structure on it. $\mathscr{S}_{\mathrm{R} 0}, \mathscr{S}_{[\mathrm{R}]_{0}}$ will denote the subspaces of $\mathscr{S}_{\mathrm{R}}, \mathscr{S}_{[\mathrm{R}]}$ consisting of all functions that vanish with all derivatives whenever at least two arguments (from $\boldsymbol{R}^{4}$ ) coincide.

If $f \in \mathscr{S}_{[\mathrm{R}]}$ and $\mathrm{R}^{\prime}$ is of the form $\pi(\mathrm{R})$ or $\pi(\mathrm{R})^{*}, \mathrm{f}_{\mathrm{R}^{\prime}}$ will denote the component of $\mathrm{f}$ in $\mathscr{S}_{\mathrm{R}^{\prime}}$.

We define a continuous involution $*$ on $\mathscr{S}_{[\mathrm{R}]}$, by

$$
\left(\mathrm{f}^{*}\right)_{\mathrm{R}}\left(x_{1}, \ldots, x_{n}\right)=\overline{\mathrm{f}_{\mathrm{R}}\left(x_{n}, \ldots, x_{1}\right)},
$$

a representation of $\mathscr{P}_{n}$ by continuous operators $\mathrm{s}_{\pi}$ for $\pi \in \mathscr{P}_{n}$ by

$$
\left(\mathrm{s}_{\pi^{\mathrm{f}}} \mathrm{f}\right)_{\mathrm{R}}\left(x_{1}, \ldots, x_{n}\right)=\sigma(\pi, \mathrm{R}) \mathrm{f}_{\pi^{-1}(\mathrm{R})}\left(x_{\pi(1)}, \ldots, x_{\pi(n)}\right)
$$

$(\sigma(\pi, \mathbf{R})$ as in Section 2$)$ and a continuous representation of inhomogeneous $S U(2) \times S U(2), \mathrm{v}(\mathrm{A}, \mathrm{B}, a)$ for $\mathrm{A}, \mathrm{B} \in S U(2), a \in \boldsymbol{R}^{4}$ by

$$
(\mathrm{v}(\mathrm{A}, \mathrm{B}, a) \mathrm{f})_{\mathrm{R}}\left(x_{1}, \ldots, x_{n}\right)=\mathrm{S}_{\mathrm{R}}^{\prime \mathrm{T}}\left(\mathrm{A}^{-1}, \mathrm{~B}^{-1}\right) \mathrm{f}_{\mathrm{R}}\left(\mathrm{L}(\mathrm{A}, \mathrm{B})^{-1}\left(x_{1}-a\right), \ldots\right)
$$

$\mathbf{S}^{\prime} \mathrm{T}$ is the transposed matrix of $\mathbf{S}^{\prime} . \mathscr{S}_{\mathrm{R}_{0}}$ and $\mathscr{S}_{[\mathrm{R}]_{0}}$ are invariant under $*, \mathrm{~s}_{\pi}$ and $\mathrm{v}(\mathrm{A}, \mathrm{B}, a) . \mathrm{v}(\mathrm{A}, \mathrm{B}, a)$ commutes with $*$ (by $(4.37,39))$ and $\mathrm{s}_{\pi}$, while $\mathrm{s}_{\pi} *=* \mathrm{~S}_{\pi^{\prime}}$ for a certain $\pi^{\prime} \in \mathscr{P}_{n}$.

By [1], the $\mathfrak{X}_{\mathrm{R}}$ define distributions (i.e. continuous linear functionals) on 
$\mathscr{S}_{\mathrm{R}_{0}}$. Let $\mathfrak{X}_{[\mathrm{R}]}$ be the corresponding distribution on $\mathscr{S}_{[\mathrm{R}] \mathrm{0}}$.

By Proposition 3 , for $\mathrm{f} \in \mathscr{S}_{[\mathrm{R}]_{0}}$,

$$
\mathfrak{X}_{[\mathrm{R}]}(\mathrm{f})=\overline{\mathfrak{X}_{[\mathrm{R}]}\left(\mathrm{f}^{*}\right)}=\mathfrak{X}_{[\mathrm{R}]}(\mathrm{v}(\mathrm{A}, \mathrm{B}, a) \mathrm{f})=\mathfrak{X}_{[\mathrm{R}]}\left(\mathrm{s}_{\pi} \mathrm{f}\right) .
$$

Proposition 4 will be proved if we can find an extension of $\mathfrak{X}_{[\mathrm{R}]}$ to $\mathscr{S}_{[\mathrm{R}]}$ (cf. [9]), satisfying (4.48) for all $\mathrm{f} \in \mathscr{S}_{[\mathrm{R}]}$. This extension will be made in three steps.

1. By the Hahn-Banach theorem and the invariance of $\mathfrak{X}_{[R]}$ under space translations, we can find a continuous linear functional, ext $\mathfrak{x}_{[\mathrm{R}]}$, on $\mathscr{S}_{[\mathrm{R}]}$, which extends $\mathfrak{X}_{[\mathrm{R}]}$ and is invariant under space translations.

2. Now let $\mu_{n}$ be normalized Haar measure on the compact group $\mathrm{G}_{n}$ $=\mathscr{P}_{n} \times S U(2) \times S U(2)$ and define, for $\mathrm{f} \in \mathscr{S}_{[\mathrm{R}]}$,

$$
\operatorname{ext}_{2}, \mathfrak{X}_{[R]}(\mathrm{f})=\operatorname{ext}_{1} \mathfrak{X}_{[R]}\left(\int_{\mathbf{G}_{n}} \mathrm{~S}_{\pi} \mathrm{v}(\mathrm{A}, \mathrm{B}, 0) \mathrm{fd} \mu_{n}(\pi, \mathrm{A}, \mathrm{B})\right) .
$$

Since the integrand is continuous on $\mathrm{G}_{n}, \operatorname{ext}_{2} \mathfrak{X}_{[\mathrm{R}]}$ is well defined. It is also continuous, linear and invariant under $\mathscr{P}_{n}$ and inhomogeneous $S U(2) \times S U(2)$. By (4.48), ext $\mathfrak{X}_{[\mathrm{R}]}$ extends $\mathfrak{X}_{[\mathrm{R}]}$.

3. Finally, for $\mathrm{f} \in \mathscr{S}_{[\mathrm{R}]}$, let

$$
\operatorname{ext}_{3} \mathfrak{X}_{[R]}(f)=\frac{1}{2}\left(\operatorname{ext}_{2} \mathfrak{X}_{[R]}(f)+\overline{\operatorname{ext}_{2} \mathfrak{X}_{[R]}\left(f^{*}\right)}\right) \text {. }
$$

This functional is continuous and linear on $\mathscr{S}_{[\mathrm{R}]}$ and an extension of $\mathfrak{X}_{[\mathrm{R}]}$. Using the commutation properties of $*, \mathrm{~s}_{\pi}$ and $\mathrm{v}(\mathrm{A}, \mathrm{B}, a)$ we find that it satisfies the relation in (4.48) for all $\mathrm{f} \in \mathscr{S}_{[\mathrm{R}]}$.

This proves Proposition 4.

Q.E.D.

In what follows we will for convenience denote ext $\mathfrak{X}_{[R]}$ by $\mathfrak{X}_{[R]}$.

(D) Constructing the Euclidean field theory; the proof of the Main Theorem.

The construction of the state space and fields given here is closely analogous to that of the Wightman reconstruction theorem ([8]).

Since we want $\Omega$ to be cyclic, we start from subspaces of the $\mathscr{S}_{\mathrm{R}}$. Let $\mathscr{L}_{\mathrm{R}}$ $\subset \mathscr{S}_{\mathrm{R}}$ be the algebraic tensor product of the $\mathscr{S}_{i_{k}}$, i.e. for $\mathrm{f} \in \mathscr{L}_{\mathrm{R}}, \mathrm{f}_{\boldsymbol{\alpha}_{i_{1}} \cdots \alpha_{i_{n}}}$ is a finite linear combination of functions of the form $\mathrm{f}_{\alpha_{i_{1}}}\left(x_{1}\right) \cdots \mathrm{g}_{\alpha_{i_{n}}}\left(x_{n}\right)$.

Let $\mathscr{L}$ be the algebraic direct sum of all the $\mathscr{L}_{\mathrm{R}}\left(\mathscr{L}_{\mathrm{R}}\right.$ for $\mathrm{R}$ empty being defined to be $\mathbb{C}$ ), i.e. each vector $\mathrm{f}$ in $\mathscr{L}$ has only a finite number of components $f_{R}$ non-zero. 
It is easy to see that $\mathrm{v}(\mathrm{A}, \mathrm{B}, a), \mathrm{s}_{\pi}$ and $*$ defined in $(\mathrm{C})$ leave $\mathscr{L}$ invariant.

On $\mathscr{L}$ we also define a multiplication, $x$, by

$$
(\mathrm{f} \times \mathrm{g})_{\mathrm{R}}\left(x_{1}, \ldots, x_{n}\right)=\sum_{\mathrm{R}=\mathrm{R}_{1} \mathrm{R}_{2}} \mathrm{f}_{\mathrm{R}_{1}}\left(x_{1}, \ldots, x_{k}\right) \mathrm{g}_{\mathrm{R}_{2}}\left(x_{k+1}, \ldots, x_{n}\right)
$$

for $\mathrm{f}, \mathrm{g} \in \mathscr{L} . \quad \mathrm{R}_{1} \mathrm{R}_{2}$ is the sequence "first $\mathrm{R}_{1}$, then $\mathrm{R}_{2}$ ".

The extended re-defined Schwinger functions $\ddot{\mathfrak{X}}_{\mathrm{R}}$ define a linear functional $\mathfrak{x}$ on $\mathscr{L}$. For $\mathrm{f}, \mathrm{g} \in \mathscr{L}$, define

$$
\langle\mathrm{f}, \mathrm{g}\rangle_{1}=\mathfrak{\mathfrak { Z }}\left(\mathrm{f}^{*} \times \mathrm{g}\right) .
$$

Since $(\mathrm{f} \times \mathrm{g})^{*}=\mathrm{g}^{*} \times \mathrm{f}^{*},\langle\cdot, \cdot\rangle_{1}$ is an inner product on $\mathscr{L}$.

Now we define the state space of the Euclidean theory

$$
\mathscr{K}=\mathscr{L} \mid\left\{\mathrm{g} \in \mathscr{L} \mid\langle\mathrm{f}, \mathrm{g}\rangle_{1}=0, \forall \mathrm{f} \in \mathscr{L}\right\} .
$$

$\langle\cdot, \cdot\rangle_{1}$ clearly defines a non-degenerate inner product on $\mathscr{K}$. It will be denoted $\langle\cdot, \cdot\rangle$.

For $\mathrm{A}, \mathrm{B} \in S U(2), a \in \boldsymbol{R}^{4}$, define $\mathrm{V}_{1}(\mathrm{~A}, \mathrm{~B}, a)$ on $\mathscr{L}$ by

$$
\left(\mathrm{V}_{1}(\mathrm{~A}, \mathrm{~B}, a) \mathrm{f}\right)_{\mathrm{R}}=\mathrm{v}(\mathrm{A}, \mathrm{B}, a) \mathrm{f}_{\mathrm{R}} \text {. }
$$

By (4.48) (for the extended $\mathfrak{\mathfrak { X }}$ ), $\mathrm{V}_{1}(\mathrm{~A}, \mathrm{~B}, a)$ is unitary w.r.t. $\langle\cdot, \cdot\rangle_{1}$, and $\Omega_{1}$ $=1 \in \boldsymbol{C}=\mathscr{L}_{\phi}$ is invariant. By unitarity, $\mathrm{V}_{1}(\mathrm{~A}, \mathrm{~B}, a)$ defines an operator $\mathrm{V}(\mathrm{A}, \mathrm{B}, a)$, unitary w.r.t. $\langle\cdot, \cdot\rangle$, on $\mathscr{K} . \mathrm{V}(\mathrm{A}, \mathrm{B}, a)$ leaves $\Omega$, the equivalence class of $\Omega_{1}$, invariant. Now, for $\mathrm{h} \in \mathscr{S}_{i}$, define ${ }_{1} \Psi^{(i)}(\mathrm{h})$ on $\mathscr{L}$ by

$$
{ }_{1} \Psi^{(i)}(\mathrm{h}) \mathrm{f}=\mathrm{h}^{(i)} \times \mathrm{f}, \quad \mathrm{f} \in \mathscr{L},
$$

where $\left(\mathrm{h}^{(i)}\right)_{\mathrm{R}}=\mathrm{h}$ if $\mathrm{R}=i$, and $=0$ if $\mathrm{R} \neq i$. By our choice of $\mathscr{L}, \Omega_{1}$ is cyclic w.r.t. the ${ }_{1} \Psi(i)$.

For $\mathrm{f}, \mathrm{g} \in \mathscr{L}$, we find

$$
\begin{aligned}
\left\langle\mathrm{f},{ }_{1} \Psi^{(i)}(\mathrm{h}) \mathrm{g}\right\rangle_{1} & =\mathfrak{X}\left(\mathrm{f}^{*} \times \mathrm{h}^{(i)} \times \mathrm{g}\right)=\mathfrak{X}\left(\left(\overline{\mathrm{h}}^{\left(i^{*}\right)} \times \mathrm{f}\right)^{*} \times \mathrm{g}\right) \\
& =\left\langle{ }_{1} \Psi^{\left(i^{*}\right)}(\overline{\mathrm{h}}) \mathrm{f}, \mathrm{g}\right\rangle_{1} .
\end{aligned}
$$

Hence ${ }_{1} \Psi^{(i)}(\mathrm{h})$ defines an operator $\Psi^{(i)}(\mathrm{h})$ on $\mathscr{K}$,

$$
\Psi^{(i)}(\mathrm{h})^{\dagger}=\Psi^{\left(i^{*}\right)}(\overline{\mathrm{h}})
$$

and $\Omega$ is cyclic in $\mathscr{K}$ w.r.t. the $\Psi^{(i)}, i \in \mathscr{I}$.

By $(4.54,55)$,

$$
\mathrm{V}_{1}(\mathrm{~A}, \mathrm{~B}, a)^{-1}{ }_{1} \Psi^{(i)}(\mathrm{h}) \mathrm{V}_{1}(\mathrm{~A}, \mathrm{~B}, a)={ }_{1} \Psi^{(i)}\left(\mathrm{v}(\mathrm{A}, \mathrm{B}, a)^{-1} \mathrm{~h}\right)
$$

The same relation holds for $\mathrm{V}, \Psi^{(i)}$ which implies 


$$
\mathrm{V}(\mathrm{A}, \mathrm{B}, a)^{-1} \Psi^{(i)}(x) \mathrm{V}(\mathrm{A}, \mathrm{B}, a)=\mathrm{S}^{\prime(i)}(\mathrm{A}, \mathrm{B}) \Psi^{(i)}\left(\mathrm{L}(\mathrm{A}, \mathrm{B})^{-1}(x-a)\right) .
$$

That the re-defined Schwinger functions are obtained as vacuum expectation values of ${ }_{1} \Psi(i)$ is clear from $(4.52,55)$. By the definition of $\langle\cdot, \cdot\rangle$ and $\Psi^{(i)}$, the same is true of the $\Psi^{(i)}$.

For the commutation properties of the $\Psi^{(i)}$, finally, note that by the symmetry of the $\mathfrak{X}_{R}$

$$
\left\langle\mathrm{f},{ }_{1} \Psi^{(i)}(\mathrm{h})_{1} \Psi^{(j)}(\mathrm{k}) \mathrm{g}\right\rangle_{1}= \pm\left\langle\mathrm{f},{ }_{1} \Psi^{(j)}(\mathrm{k})_{1} \Psi^{(i)}(\mathrm{h}) \mathrm{g}\right\rangle_{1}
$$

for $\mathrm{f}, \mathrm{g} \in \mathscr{L}, i, j \in \mathscr{I}$ and $\mathrm{h} \in \mathscr{S}_{i}, \mathrm{k} \in \mathscr{S}_{j}$. The sign depends on the boson or fermion nature of the indices $i$ and $j$. (4.60) implies the desired commutation properties for the $\Psi^{(i)}$.

This proves our Main Theorem. $\quad$ Q.E.D.

\section{Acknowledgements}

I am very grateful to Professor $\mathrm{H}$. Araki for hospitality during my time at the Research Institute for Mathematical Sciences of Kyoto University, for suggesting me to study the use of an indefinite inner product state space for Euclidean fields with spin and for many helpful suggestions during the course of this investigation as well as during the preparation of this manuscript.

\section{References}

[1] Osterwalder, K. and Schrader, R., Commun. Math. Phys., 31 (1973), 83-112.

[2] Osterwalder, K. and Schrader, R., Commun. Math. Phys., 42 (1975), 281-305.

[ 3 ] Nelson, E., J. Functional Analysis, 12 (1973), 97-112.

[4] Gross, L., Euclidean Fermion Fields, Les méthodes mathématiques de la théorie quantique des champs (Colloques Internationaux C. N .R. S. $\mathrm{N}^{\circ}$ 248), Paris, 1976, 131146.

[5] Osterwalder, K. and Schrader, R., Helv. Phys. Acta, 46 (1973), 277-302.

[6] Özkaynak, H., Euclidean Fields for Massive Particles of Arbitrary Spin, Harvard University Preprint, 1974.

[7] Fröhlich, J. and Osterwalder, K., Is there a Euclidean Field Theory for Fermions, Harvard University Preprint, 1974.

[ 8 ] Streater, R. F. and Wightman, A. S., PCT, Spin and Statistics, and All That, second printing, with additions and corrections, Reading, Benjamin/Cummings, Massachusetts, 1978.

[9] Challifour, J. L. and Slinker, S. P., Commun. Math. Phys., 43 (1975), 41-58.

[10] Borchers, H. J. and Yngvason, J., Commun. Math. Phys., 43 (1975), 255-271.

[11] Simon, B., Helv. Phys. Acta, 46 (1973), 686-696. 
[12] Borchers, H. J. and Yngvason, J., Commun. Math. Phys., 47 (1976), 197-213.

[13] Feinberg, G. and Weinberg, S., Nuovo Cimento, 14, (1959), 571-592.

[14] Bognár, J., Indefinite Inner Product Spaces, Springer, Berlin, Heidelberg, New York, 1974.

[15] Yngvason, J., Commun. Math. Phys., 34 (1973), 315-333.

[16] Wilde, I. F., J. Functional Analysis, 15 (1974), 12-21.

[17] Palmer, J., J. Functional Analysis, 36 (1980), 287-312. 